\title{
Association of Common Genetic Variants with Diabetes and Metabolic Syndrome Related Traits in the Arizona Insulin Resistance Registry: A Focus on Mexican American Families in the Southwest
}

\author{
Jacob DeMenna ${ }^{a}$ Sobha Puppala ${ }^{b}$ Geetha Chittoor ${ }^{b}$ Jennifer Schneider ${ }^{b}$ \\ Joon Young Kim ${ }^{c}$ Gabriel Q. Shaibi ${ }^{d}$ e Lawrence J. Mandarino ${ }^{\mathrm{a}, \mathrm{e}}$ \\ Ravindranath Duggiralab Dawn K. Coletta ${ }^{a, e, f}$ \\ a School of Life Sciences, Arizona State University, Tempe, Ariz., b Department of Genetics, Texas Biomedical \\ Research Institute, San Antonio, Tex., 'Kinesiology Program, School of Nutrition and Health Promotion, Arizona \\ State University, Phoenix, Ariz., ' ${ }^{d}$ College of Nursing and Health Innovation, Arizona State University, Tempe, Ariz., \\ ${ }^{\mathrm{e}}$ Mayo Clinic in Arizona, Scottsdale, Ariz., and ${ }^{\mathrm{f} D e p a r t m e n t}$ of Basic Medical Sciences, University of Arizona College \\ of Medicine, Phoenix, Ariz., USA
}

\section{Key Words}

Arizona Insulin Resistance registry · Mexican Americans · SNP genotyping · Diabetes $\cdot$ Metabolic syndrome $\cdot$ GWAS

\footnotetext{
Abstract

Background/Aims: The increased occurrence of type 2 diabetes and its clinical correlates is a global public health issue, and there are continued efforts to find its genetic determinant across ethnically diverse populations. The aims of this study were to determine the heritability of diabetes and metabolic syndrome phenotypes in the Arizona Insulin Resistance (AIR) registry and to perform an association analysis of common single nucleotide polymorphisms (SNPs) identified by GWAS with these traits. All study participants were Mexican Americans from the AIR registry. Methods: Metabolic, anthropometric, demographic and medical history information was obtained on the 667 individuals enrolled in the registry. Results: The heritability estimates were moderate to high in magnitude and significant, indicating that the
}

AIR registry is well suited for the identification of genetic factors contributing to diabetes and the metabolic syndrome. From the 30 GWAS genes selected (some genes were represented by multiple SNPs), 20 SNPs exhibited associations with one or more of the diabetes related traits with nominal significance ( $p \leq 0.05$ ). In addition, 25 SNPs were nominally significantly associated with one or more of the metabolic phenotypes tested ( $p \leq 0.05)$. Most notably, 5 SNPs from 5 genes [body mass index (BMI), hip circumference: rs3751812/ FTO; fasting plasma glucose, hemoglobin A1c: rs4607517/ GCK; very-low-density lipoprotein: rs10830963/MTNR1B; BMI: rs13266634/SLC30A8, and total cholesterol, low-density lipoprotein: rs7578597/THADA] were significantly associated with obesity, glycemic, and lipid phenotypes when using the multiple testing significance threshold of 0.0015 . Conclusion: These findings extend previous work on Mexican Americans to suggest that metabolic disease is strongly influenced by genetic background in this high-risk population.

(c) 2014 S. Karger AG, Basel

\section{KARGER}

E-Mail karger@karger.com

www.karger.com/hhe
(C) 2014 S. Karger AG, Basel

0001-5652/14/0781-0047\$39.50/0
Dawn K. Coletta, PhD

School of Life Sciences

Arizona State University

PO Box 874501, Tempe, AZ 85287-4501 (USA)

E-Mail dawn.coletta@asu.edu 


\section{Introduction}

The metabolic syndrome represents a clustering of type 2 diabetes risk factors, including hyperglycemia, insulin resistance, hypertension, dyslipidemia, obesity and endothelial dysfunction $[1,2]$. Over the past three decades, the global prevalences of obesity, type 2 diabetes and the metabolic syndrome have rapidly and relentlessly increased. In the United States, 35.7\% of adults are obese [3], 8.3\% have type 2 diabetes and as many as 39\% are estimated to have the metabolic syndrome [4]. Moreover, these prevalence rates are even higher among certain populations, including Mexican Americans.

Many studies have identified environmental factors, including physical inactivity and caloric excess, which contribute to the pathogenesis of type 2 diabetes and the metabolic syndrome [5]. Likewise, there is much evidence to demonstrate that genetics plays an important role in these diseases [6-13]. To date, over 70 susceptibility loci have been associated with type 2 diabetes and related metabolic traits, including TCF7L2, SLC2A2, IGF2BP2, CDKAL1, FTO, GCK, JAZF1, THADA, $\mathrm{NOTCH} 2, \mathrm{CDKN} 2 \mathrm{~A} / \mathrm{B}$ and MADD, with the majority of those identified by GWAS [6, 7, 13-20]. However, the majority of these studies were performed on European populations and much less is known about the genetic influences on metabolic disease in Mexican Americans.

Recently, a GWAS and meta-analysis performed on Mexican Americans from Starr County, Texas, and Mexico City identified associations with previously reported type 2 diabetes susceptibility single nucleotide polymorphisms (SNPs) as well as novel loci $[21,22]$. Specifically, the GWAS in Mexican Americans identified a novel locus (C14orf70) and provided suggestive associations for other previously reported loci (HNF1A, KCNQ1, IGF2BP2 and CDKN2A/CDKN2B) [22]. In addition, 24 common genetic variants previously associated with type 2 diabetes were genotyped in a Mexican Mestizo population [23]. In that study, the authors confirmed that the associations previously identified in European populations, including rs13266634 (SLC30A8), rs7923837 (HHEX), rs10811661 (CDKN2A/2B), rs4402960 (IGF2BP2), rs12779790 (CDC123/CAMK1D), and rs2237892 (KCNQ1) were also present in the Mexican Mestizo population [23]. The transcription factor-7-like 2 (TCF7L2) has been studied in Mexican Americans from San Antonio, Texas, and it has been demonstrated that variation in the TCF7L2 genomic region may affect the risk for type 2 diabetes in this population, but the risk may be lower than in the nonHispanic white population [24].
Table 1. Distribution of relative pairs by category in the AIR registry families

\begin{tabular}{llr}
\hline & $\begin{array}{l}\text { Relationship } \\
\text { coefficient* }\end{array}$ & Pairs, n \\
\hline Parent-offspring & 0.50 & 229 \\
Siblings & 0.50 & 104 \\
Grandparent-grandchild & 0.25 & 34 \\
Avuncular & 0.25 & 150 \\
Half siblings & 0.25 & 55 \\
Great grandparent-great grandchild & 0.125 & 3 \\
Grand avuncular & 0.1250 & 6 \\
Half avuncular & 0.1250 & 4 \\
First cousins & 0.1250 & 114 \\
Half grand avuncular & 0.0625 & 1 \\
First cousins, once removed & 0.0625 & 15 \\
Half first cousins & 0.0625 & 5 \\
Half first cousins, once removed & 0.03125 & 2 \\
Second cousins & 0.03125 & 1 \\
\hline Total & & 723 \\
\hline
\end{tabular}

* The relationship coefficient is twice the coefficient of kinship of two individuals.

Given the limited available data on how genetic factors contribute to metabolic disease among Mexican Americans, we developed the Arizona Insulin Resistance (AIR) registry as a mechanism to facilitate biomedical and genetic research in this high-risk population. A description of the registry and the goals of this study have been discussed elsewhere [25]. The present study describes the initial heritability estimates for type 2 diabetes and metabolic syndrome phenotypes in the AIR registry. Additionally, we determined if SNP associations with type 2 diabetes and related traits selected from previously published GWAS studies were also present in this population.

\section{Subjects and Methods}

\section{AIR Registry}

All study participants were Mexican Americans from the AIR registry, which has been described elsewhere [25]. Briefly, of the 667 participants enrolled in the study, 353 were distributed across 92 families from the AIR registry. The remaining 314 participants represented single unrelated individuals, who were considered for the analysis because they contribute to the evaluation of covariate effects. The 353 participants from 92 families generated 723 relative pairs that were distributed across 14 relative-pair categories (table 1). Metabolic, anthropometric, demographic and medical history information was obtained on the 667 individuals (aged
DeMenna et al. 
8-83 years old) enrolled in the study (table 2). The Institutional Review Board of Arizona State University approved all procedures, and all subjects gave informed written consent before their participation.

Blood samples were obtained after a 12-hour fast for the assessment of various phenotypes, including glucose, insulin, triglycerides, total cholesterol (Chol), high-density lipoprotein cholesterol (HDL), low-density lipoprotein cholesterol (LDL) and very-lowdensity lipoprotein cholesterol (VLDL). In addition, all participants $\geq 12$ years of age $(n=641)$ underwent a 2 -hour oral glucose tolerance test (2hOGTT), where a solution containing $1.75 \mathrm{~g} / \mathrm{kg}$ dextrose (up to $75 \mathrm{~g}$ ) was ingested and venous blood samples were obtained at 30,60, 90, and 120 min for the determination of glucose and insulin concentrations. Plasma glucose was measured by the glucose oxidase method using a YSI 2300 STAT plus (YSI INC., Yellow Sprigs, Ohio, USA), and insulin was measured in duplicate by an enzyme-linked immunosorbent assay (ALPCO Diagnostics, Windham, N.H., USA). All other laboratory tests were performed by a Clinical Laboratory Improvement Amendments-certified commercial laboratory (Sonora Quest Laboratories, Phoenix, Ariz., USA).

\section{SNP Selection and Genotyping}

We isolated genomic DNA from whole blood using the PAXgene Blood DNA procedure per the manufacturer's instructions (Qiagen, Calif., USA). A total of 40 SNPs representing 30 GWASidentified genes/loci were selected from published studies that previously reported an association with type 2 diabetes and related traits (online suppl. table 1; for all online suppl. material, see www. karger.com/doi/10.1159/000363411). The SNPs were selected from candidate genes and most of the first-generation GWAS type 2 diabetes risk variants reported to assess their transferability and to examine their relevance to various metabolic syndrome related traits in a Mexican American population. Additional SNPs in the FTO and TCF7L2 genes were chosen based on multiple variants in those genes associated with type 2 diabetes or related phenotypes. The 40 SNPs were genotyped in the AIR registry subjects.

We obtained SNPs by the Assay-by-Design service (Applied Biosystems, Calif., USA), and they were genotyped as previously described [1]. Briefly, in a 384-well plate, $2 \mu$ of purified genomic DNA $(2 \mathrm{ng} / \mu \mathrm{l})$ were incubated with primers and probes with the SNP of interest $(0.09 \mu \mathrm{l}), 3.5 \mu \mathrm{l}$ of TaqMan Universal Polymerase Chain Reaction Master Mix-No AmpErase UNG and $1.14 \mu \mathrm{l}$ of distilled water. Samples were polymerase-chain-reaction-amplified on the Applied Biosystems 9700HT Thermal Cycler under the following conditions: denatured for $10 \mathrm{~min}$ at $95^{\circ} \mathrm{C}$, denatured, annealed, and extended for 40 cycles of $15 \mathrm{~s}$ at $92^{\circ} \mathrm{C}$ and $1 \mathrm{~min}$ at $60^{\circ} \mathrm{C}$. The 384 -well microplates were scanned for fluorescence emission using a 7900HT sequence detector (Applied Biosystems), and alleles were scored using the allelic discrimination software Sequence Detection System v2.3 (Applied Biosystems). For all SNPs genotyped, our mean rate of success for genotyping was $>99 \%$.

\section{Phenotypes for Genetic Analysis}

A total of 21 diabetes and metabolic phenotypes, including indices of insulin action and secretion, were used for the analysis: body mass index (BMI), waist circumference (WC), hip circumference (HC), total cholesterol (Chol), HDL, LDL, VLDL, triglyceride (TG), systolic blood pressure (SBP), diastolic blood pressure
Table 2. Characteristics of the AIR registry subjects

\begin{tabular}{llc}
\hline Female & $(\mathrm{n}=667)$ & 61 \\
Age, years & $(\mathrm{n}=667)$ & $31.7 \pm 13.4$ \\
BMI & $(\mathrm{n}=666)$ & $28.9 \pm 7.2$ \\
WC, cm & $(\mathrm{n}=661)$ & $95.4 \pm 16.4$ \\
HC, cm & $(\mathrm{n}=661)$ & $106.2 \pm 13.6$ \\
Chol, mg/dl & $(\mathrm{n}=660)$ & $169.9 \pm 43.4$ \\
Triglycerides, mg/dl & $(\mathrm{n}=660)$ & $132.5 \pm 104.6$ \\
HDL, mg/dl & $(\mathrm{n}=660)$ & $44.5 \pm 11.2$ \\
LDL, mg/dl & $(\mathrm{n}=660)$ & $102.5 \pm 29.6$ \\
VLDL, mg/dl & $(\mathrm{n}=646)$ & $20.6 \pm 11.0$ \\
SBP, mm Hg & $(\mathrm{n}=666)$ & $118.3 \pm 15.3$ \\
DBP, mm Hg & $(\mathrm{n}=666)$ & $74.5 \pm 10.1$ \\
ALT, U/l & $(\mathrm{n}=656)$ & $26.2 \pm 21.0$ \\
AST, U/l & $(\mathrm{n}=656)$ & $24.4 \pm 12.4$ \\
HbAlc, \%* & $(\mathrm{n}=580)$ & $5.6 \pm 0.4$ \\
FPI, $\mu I U / m)^{*}$ & $(\mathrm{n}=496)$ & $9.6 \pm 8.5$ \\
HOMA IR* & $(\mathrm{n}=496)$ & $2.2 \pm 2.0$ \\
Matsuda index* & $(\mathrm{n}=471)$ & $5.9 \pm 5.5$ \\
Disposition index* & $(\mathrm{n}=471)$ & $8.5 \pm 31.1$ \\
FPG, mg/dl* & $(\mathrm{n}=574)$ & $91.7 \pm 7.5$ \\
2hOGTT, mg/dl* & $(\mathrm{n}=551)$ & $121.8 \pm 30.3$ \\
Diabetes status & $(\mathrm{n}=628)$ & 12.3 \\
Prediabetes status* & $(\mathrm{n}=551)$ & 34.0 \\
& & \\
\hline
\end{tabular}

Values are percentages or means \pm SD. * Data from non-diabetics were used.

(DBP), fasting plasma glucose (FPG), 2hOGTT, hemoglobin A1c (HbAlc), fasting plasma insulin (FPI), homeostasis model assessment for insulin resistance (HOMA IR), Matsuda index, disposition index, prediabetes status, diabetes status, alanine aminotransferase (ALT) and aspartate aminotransferase (AST). The formulas and references of the indices of insulin action, secretion and $\beta$-cell function, which are derived from the OGTT, have been described previously [26]. To normalize the trait distributions for genetic analyses, BMI, WC, TG, VLDL, SBP, ALT, AST and Matsuda index were log transformed, and HC, FPG, 2hOGTT, HbA1c, FPI, HOMA IR and disposition index were transformed using inverse normalization.

\section{Statistical Genetic Analysis}

We used a variance components (VC) approach as implemented in the program SOLAR to estimate the heritabilities of quantitative or continuous (e.g. BMI) and qualitative or dichotomous (e.g. diabetes status) traits (Sequential Oligogenic Linkage Analysis Routines (SOLAR); http://www.txbiomed.org/solar) [27]. To determine whether the heritability of a given phenotype was significant ( $\mathrm{p} \leq 0.05)$, a likelihood-ratio test was used. Each trait-specific genetic analysis determined heritability after accounting for covariate influences (e.g. age and sex), and the covariates in our models varied depending on the phenotype tested. Specifically, covariates such as age, sex, age $e^{2}$, age $\times$ sex, and age $^{2} \times$ sex were included in all analyses if found to be significant ( $\mathrm{p} \leq 0.05)$. For the purpose 
of discussion, we used the term 'age and sex terms' to refer to the significant covariates of age, sex and their higher order terms and interactions. For BMI, WC and HC, we adjusted for the covariate effects of age and sex terms and diabetes. For Chol, TG, HDL, LDL and VLDL traits, in addition to the age and sex terms and diabetes covariate effects, we also accounted for lipid medication. For SBP and DBP, in addition to age and sex terms and diabetes covariate effects, we also adjusted for hypertension medication. For ALT and AST, we accounted for the covariate effects of age and sex terms. For HbA1c, FPI, HOMA IR, Matsuda index, disposition index, FPG, 2hOGTT and prediabetes status phenotypes, we used data only from non-diabetics and accounted for covariate effects of age and sex terms only. Finally, for diabetes status, we adjusted for age and sex terms and BMI.

We performed an association analysis using the measured genotype approach (MGA) within the VC analytical framework as in SOLAR for both continuous and dichotomous traits [27, 28]. This approach allowed us to account for the non-independence among family members by incorporating a residual polygenic component. In a given model, VCs are modeled as random effects (e.g. additive genetic effects and random environmental effects), and the mean effects of the measured covariates (e.g. age and sex) are modeled as fixed effects on the trait mean. In MGA, generally, the marker genotypes are incorporated in the mean effects model as a measured covariate assuming additivity of allelic effects [28]. The VCs, the association parameters, and the other covariate effects (e.g. age and sex terms) were estimated simultaneously by maximum likelihood techniques. We considered the same covariates that were used to determine heritabilities. We tested the hypothesis of no association by comparing the likelihood of a model in which the effect of the measured genotype was estimated with a model where the effect of measured genotype was fixed at zero. Before performing MGA, using SOLAR, the quantitative transmission disequilibrium test (QTDT) was performed to evaluate population stratification, using the notation of within (w) and between (b) family components of association [29]. Using likelihood ratio tests, a significant difference ( $p \leq 0.05$ ) between the $b$ and $w$ parameters can be considered as evidence of population stratification. If the presence of population stratification was observed, the QTDT procedure was employed to assess association using within family information, which was robust to population stratification.

For the purposes of exploring the association of the GWASidentified diabetes susceptibility variants with variation in diabetes and metabolic traits in Mexican Americans in the AIR registry, we used a nominal $\mathrm{p} \leq 0.05$ as our initial threshold for statistical significance. However, to address the issue of multiple testing, given the linkage disequilibrium (LD) patterns among some of the examined SNPs, an effective number of SNPs was obtained using the method of $\mathrm{Li}$ and Ji [30] to derive a p value adjusted for multiple testing. The effective number of SNPs (i.e. 39 SNP association analyses) in the data set was 33, and the required significance threshold was 0.0015 . No additional attempts to correct for multiple testing were made, since correcting for multiple testing with regard to non-independence among the examined phenotypes/models is not straightforward. The program SOLAR was used to calculate the allele frequencies, to test deviations from Hardy-Weinberg Equilibrium (HWE) and to estimate LD between SNP pairs using $r^{2}$ values.
Table 3. Heritability estimates for diabetes and metabolic phenotypes

\begin{tabular}{|c|c|c|}
\hline & Heritability \pm SE (\%) & $\mathrm{p}$ value \\
\hline $\mathrm{BMI}^{\mathrm{a}}$ & $48 \pm 12$ & $2.2 \times 10^{-5}$ \\
\hline $\mathrm{WC}^{\mathrm{a}}$ & $46 \pm 10$ & $1.1 \times 10^{-5}$ \\
\hline $\mathrm{HC}^{\mathrm{b}}$ & $56 \pm 12$ & $3.5 \times 10^{-6}$ \\
\hline Chol & $62 \pm 13$ & $5.1 \times 10^{-6}$ \\
\hline Triglycerides $^{\mathrm{a}}$ & $60 \pm 12$ & $1.0 \times 10^{-6}$ \\
\hline HDL & $75 \pm 11$ & $1.0 \times 10^{-11}$ \\
\hline LDL & $41 \pm 17$ & 0.0071 \\
\hline VLDL $^{a}$ & $46 \pm 13$ & 0.0001 \\
\hline $\mathrm{SBP}^{\mathrm{a}}$ & $30 \pm 13$ & 0.0077 \\
\hline DBP & $11 \pm 12$ & 0.1650 \\
\hline $\mathrm{ALT}^{\mathrm{a}}$ & $48 \pm 17$ & 0.0023 \\
\hline $\mathrm{AST}^{\mathrm{a}}$ & $45 \pm 16$ & 0.0014 \\
\hline $\mathrm{HbA} 1 c^{b}$ & $52 \pm 10$ & $2.0 \times 10^{-7}$ \\
\hline FPI $^{\mathrm{b}}$ & $49 \pm 13$ & 0.0001 \\
\hline HOMA IR ${ }^{b}$ & $50 \pm 13$ & $9.5 \times 10^{-5}$ \\
\hline Matsuda index ${ }^{a}$ & $47 \pm 14$ & 0.0005 \\
\hline Disposition index ${ }^{b}$ & $36 \pm 15$ & 0.0105 \\
\hline $\mathrm{FPG}^{\mathrm{b}}$ & $15 \pm 16$ & 0.1591 \\
\hline 2 hOGTT $^{b}$ & $16 \pm 18$ & 0.1830 \\
\hline Diabetes status & 100 & 0.0456 \\
\hline Prediabetes status & $39 \pm 24$ & 0.0673 \\
\hline
\end{tabular}

a Traits were $\log$ transformed for genetic analyses. ${ }^{\mathrm{b}}$ Traits were inverse normalized for genetic analyses.

\section{Results}

The clinical characteristics of the AIR registry participants are shown in table 2. The available trait-specific sample sizes ranged from 471 (Matsuda and disposition indices; non-diabetic individuals only) to 666 (BMI, SBP and DBP; all individuals, i.e. diabetic and non-diabetic individuals). Thus, the genetic analyses for the heritability and association assessments used all available traitspecific data. Heritability estimates for the 21 diabetes and metabolic phenotypes were determined using SOLAR and were moderate to high in magnitude (range $0.30-1.00)$ and significant. All of the phenotypes measured were significant with the exception of DBP, FPG, 2hOGTT and prediabetes status (table 3). Although the heritability estimates of DBP, FPG, 2hOGTT and prediabetes status were not significant, they were within the range of those published in previous studies [31].

We genotyped 40 common SNPs (representing 30 genes/loci), and 39 of these were polymorphic and in HWE ( $\mathrm{p} \leq 0.01$ ). The marker rs1801282, which lies in the PPARG gene, was not in HWE and was discarded from subsequent analysis. Minor allele frequencies of the 39 
Table 4. Allele frequency distribution of SNPs $(n=39)$ studied in the AIR registry

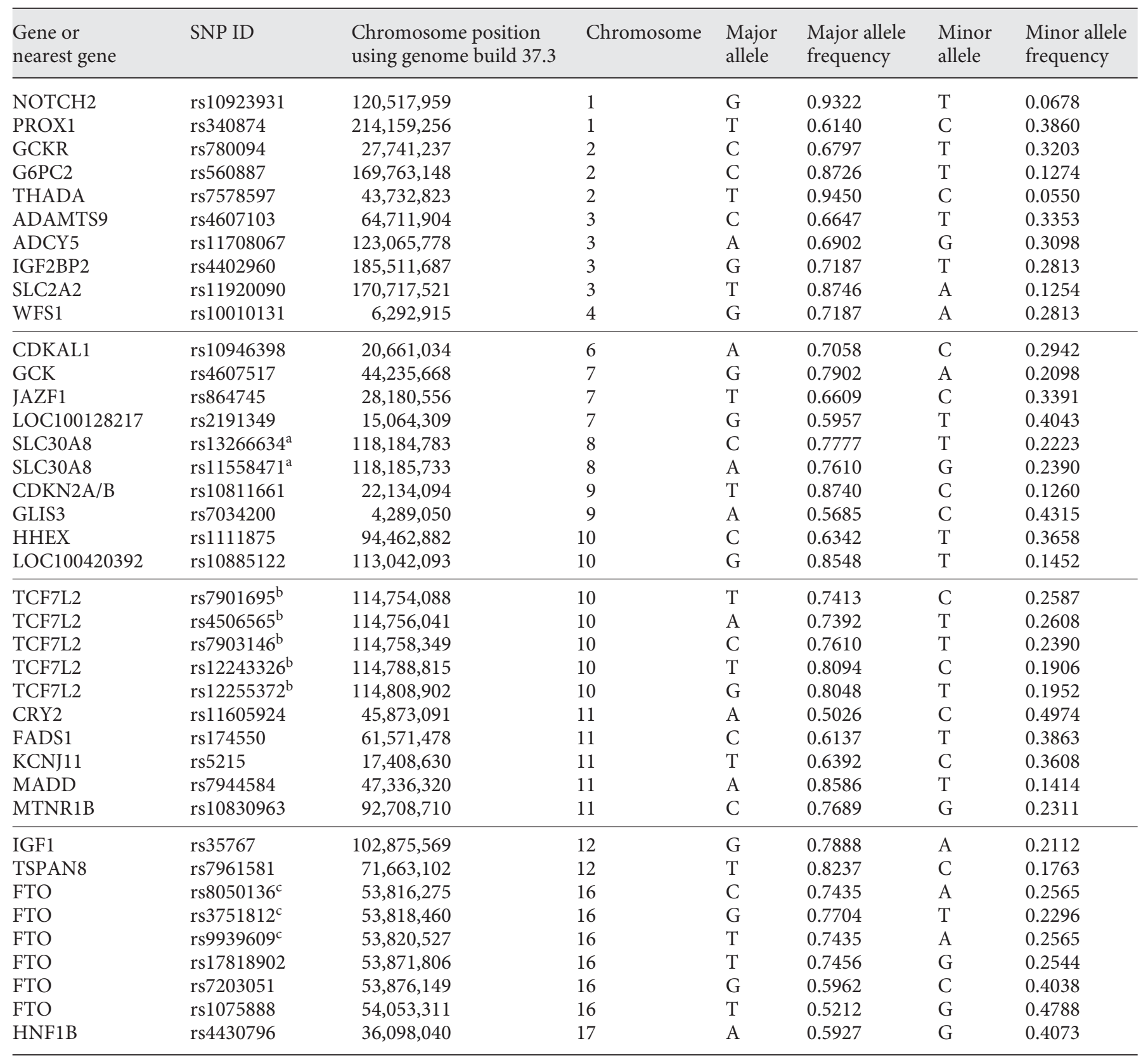

a Two SNPs in the SLC30A8 gene are in strong LD with $\mathrm{r}^{2}=0.90 .{ }^{\mathrm{b}}$ Five SNPs in the TCF7L2 gene are in strong LD with $\mathrm{r}^{2}$ ranging from 0.86 to 0.99 . ${ }^{c}$ Three SNPs in the FTO gene are in strong LD with $\mathrm{r}^{2}$ ranging from 0.87 to 0.98 .

SNPs that were in HWE ranged from approximately 0.055 to $0.497 \%$ (table 4), indicating that the SNPs were common in the AIR registry. There were 5 SNPs (rs7901695, rs4506565, rs7903146, rs12243326, rs12255372) in TCF7L2 that were in strong LD with $\mathrm{r}^{2}$ ranging from 0.86 to 0.99 (table 4 ). Similarly, there were 3 SNPs (rs3751812, rs9939609, rs8050136) in FTO that were in strong LD with $\mathrm{r}^{2}$ ranging from 0.87 to 0.98 (table 4). Likewise, 2 SNPs (rs13266634, rs11558471) in the SLC30A8 gene were in strong LD with $\mathrm{r}^{2}=0.90$ (table 4 ).

The associations of common SNPs with diabetes and metabolic syndrome related phenotypes are shown in ta- 
Table 5. Significant associations of common SNPs with diabetes related phenotypes

\begin{tabular}{|c|c|c|c|c|c|c|c|c|c|c|}
\hline ADCY5 & rs11708067 & $0.0031^{*}$ & 0.0306 & & & & & & 0.0473 & 0.0447 \\
\hline CRY2 & rs11605924 & 0.0163 & & & & & & & & \\
\hline FTO & rs8050136 & & & & 0.0210 & 0.0284 & & & & \\
\hline FTO & rs3751812 & & & & 0.0085 & 0.0088 & & & & \\
\hline FTO & rs17818902 & & & $0.0338^{*}$ & & & & & & \\
\hline G6PC2 & rs560887 & & 0.0049 & & 0.0213 & 0.0115 & & & & \\
\hline GCK & rs4607517 & $4.0 \times 10^{-5}$ & 0.0004 & 0.0147 & & & & & & 0.0037 \\
\hline GLIS3 & rs7034200 & & & & & & & & & \\
\hline IGF1 & rs35767 & & & & & & & & & $0.0229^{*}$ \\
\hline JAZF1 & rs864745 & & & & & & & & & \\
\hline TCF7L2 & rs7903146 & & & & 0.0443 & & & & & \\
\hline TCF7L2 & rs12243326 & & 0.0474 & & & & & & & \\
\hline TCF7L2 & rs12255372 & & 0.0450 & & & & & & & \\
\hline$\overline{\mathrm{THADA}}$ & rs7578597 & & & & & & & 0.0276 & & $0.0358^{*}$ \\
\hline
\end{tabular}

Both corrected ( $\mathrm{p} \leq 0.0015$; in bold) and uncorrected $(\mathrm{p} \leq 0.05) \mathrm{p}$ values for multiple testing by trait are shown, $\mathrm{p}$ values are nominal and from MGA; * $\mathrm{p}$ values are from QTDT because of the presence of population stratification.

bles 5 and 6, respectively. The nominal p values related to association analyses shown in these tables are uncorrected for multiple testing. However, all $\mathrm{p}$ values shown in bold are those corrected for multiple testing, which met the required significance threshold of 0.0015 . As reported in tables 5 and 6 , some of the tests to determine the presence of population stratification could not be rejected, hence the association findings from the QTDT procedure were reported.

There were 20 SNPs that exhibited nominal associations with one or more of the diabetes and related phenotypes tested (table 5). Since the HbA1c, FPI, HOMA IR, Matsuda index, disposition index, FPG and 2hOGTT analyses used data from non-diabetic individuals, which included those with prediabetes, we reanalyzed these trait associations after excluding the prediabetic individuals. Given the fact that the sample sizes were decreased, as expected, several of the association findings of these traits have been attenuated, some even becoming nonsignificant (data not shown). These data reveal that the examined diabetes risk variants appear to be potential markers for assessing the increased risk of metabolic abnormalities associated with a prediabetes condition.
There were 25 SNPs that exhibited nominal associations with one or more of the metabolic phenotypes tested (table 6). However, as reported in tables 5 and 6, only 5 SNPs from 5 genes (BMI, HC: rs3751812/FTO; FPG, HbA1c: rs4607517/GCK; VLDL: rs10830963/MTNR1B; BMI: rs13266634/SLC30A8, and Chol, LDL: rs7578597/ THADA) were significantly associated with obesity, glycemic and lipid phenotypes when using the required significance threshold of 0.0015 .

The genotype class specific mean values for the quantitative traits related to diabetes and the metabolic syndrome that were associated with a $\mathrm{p} \leq 0.01$ (i.e. uncorrected for multiple testing), including the 8 significantly associated phenotypes ( $\mathrm{p} \leq 0.0015)$, are shown in table 7 . Of the 34 significant associations, 17 were found to replicate the same association direction (i.e. same risk allele) as the one identified in the original GWAS or candidate gene studies (online suppl. table 1). We observed similar $\mathrm{p}$ value associations for the TCF7L2, FTO and SLC30A8 SNPs that were in strong LD and, as such, we have shown the genotype-class-specific mean values for rs3751812/ FTO, rs7903146/TCF7L2 and rs13266634/SLC30A8. As reported above, rs3751812 (FTO), rs4607517/GCK, 
Table 6. Significant associations of common SNPs with metabolic syndrome phenotypes

\begin{tabular}{|c|c|c|c|c|c|c|c|c|c|c|c|c|c|}
\hline ADCY5 & rs11708067 & 0.0359 & 0.0395 & 0.0477 & & & & & & & & & \\
\hline$\overline{\mathrm{CRY} 2}$ & rs11605924 & & & & & & & & 0.0388 & & & & $0.0308^{*}$ \\
\hline$\overline{\text { FTO }}$ & rs8050136 & 0.0096 & 0.0121 & 0.0031 & & & & & & & & & \\
\hline$\overline{\text { FTO }}$ & rs3751812 & 0.0010 & 0.0023 & 0.0002 & & & & & & & & & \\
\hline FTO & rs9939609 & 0.0100 & 0.0216 & 0.0042 & & & & & & & & & \\
\hline GLIS3 & rs7034200 & & & & & & & 0.0361 & & 0.0175 & 0.0391 & & \\
\hline HNF1B & rs4430796 & 0.0278 & & & & & & 0.0175 & & 0.0493 & & & \\
\hline$\overline{\text { IGF2BP2 }}$ & rs4402960 & & & & & 0.0281 & & & & & & & \\
\hline JAZF1 & rs864745 & & & & & & 0.0420 & & & & & & 0.0440 \\
\hline LOC100128217 & rs2191349 & & & & & & & & & & 0.0532 & & \\
\hline SLC30A8 & rs13266634 & 0.0013* & $0.0031^{*}$ & $0.0060^{*}$ & $0.0229^{*}$ & & & & & $0.0492^{*}$ & 0.0455 & & \\
\hline SLC30A8 & rs11558471 & $0.0028^{*}$ & $0.0127^{*}$ & $0.0154^{*}$ & $0.0182^{*}$ & & & & & & & & \\
\hline TCF7L2 & rs7901695 & $0.0311^{*}$ & & & & 0.0158 & & $0.0263^{*}$ & & 0.0168 & $0.0057^{*}$ & $0.0035^{*}$ & \\
\hline TCF7L2 & rs4506565 & $0.0406^{*}$ & & & & 0.0089 & & $0.0286^{*}$ & & 0.0189 & $0.0064^{*}$ & $0.0036^{*}$ & \\
\hline TCF7L2 & rs7903146 & $0.0102^{*}$ & $0.0151^{*}$ & & & 0.0148 & & $0.0042^{*}$ & & 0.0068 & 0.0027 & $0.0049^{*}$ & \\
\hline TCF7L2 & rs12243326 & $0.0250^{*}$ & & & & & & $0.0131^{*}$ & & $0.0076^{*}$ & $0.0032^{*}$ & & \\
\hline TCF7L2 & rs12255372 & & & & & & & $0.0142^{*}$ & & & $0.0165^{*}$ & & \\
\hline THADA & rs7578597 & & & & & $0.0048^{*}$ & $8.8 \times 10^{-5 *}$ & & $0.0003^{*}$ & $0.0165^{*}$ & $0.0285^{*}$ & & 0.0243 \\
\hline
\end{tabular}

Both corrected ( $\mathrm{p} \leq 0.0015$; in bold) and uncorrected $(\mathrm{p} \leq 0.05) \mathrm{p}$ values for multiple testing by trait are shown, $\mathrm{p}$ values are nominal and from MGA; * $\mathrm{p}$ values are from QTDT because of the presence of population stratification.

rs10830963 (MTNR1B), rs13266634 (SLC30A8) and rs7578597 (THADA) were significantly associated with one or more diabetes and/or metabolic phenotypes ( $\mathrm{p} \leq$ $0.0015)$. As shown in table 5 , none of the associations with diabetes and prediabetes reached the required significance threshold, although 2 variants exhibited associations with a $\mathrm{p} \leq 0.01$ (i.e. uncorrected for multiple testing) as follows: $\mathrm{rs} 10811661 / \mathrm{CDKN} 2 \mathrm{~A} / \mathrm{B}, \beta \mathrm{SNP}=-0.012 \pm$ $0.002, \mathrm{p}=0.0115$ and $\mathrm{rs} 4607517 / \mathrm{GCK}, \beta \mathrm{SNP}=0.292 \pm$ $0.101, \mathrm{p}=0.0037$. The direction of the prediabetes associations was consistent compared in these SNP associations with glucose, HbA1C and Matsuda index (table 7).

\section{Discussion}

This study examines for the first time the genetic contributions to diabetes and metabolic traits in the AIR registry sample. The AIR registry is composed of Mexican American individuals residing in the area of Phoenix, Ar- izona, and was extensively phenotyped for type 2 diabetes and metabolic syndrome measures. The prevalence of diabetes in our population was $12.3 \%$, which was above the $8.3 \%$ of the general population $[32,33]$. Moreover, we observed that $34 \%$ of the participants were classified with prediabetes, which was similar to national averages that showed a $36 \%$ prevalence of prediabetes in Mexican American adults in the United States [33].

Although most of the heritability estimates of the phenotypes examined in the AIR registry appear to be consistent with previously published studies [31], it is possible that they may have been inflated, since shared environmental influences were not accounted for in our analyses. Also, it is possible that heritability can be age dependent, which may be underestimated when age dependence is present. The heritability estimates of DBP, FPG, 2hOGTT and prediabetes did not reach statistical significance in our study. Perhaps DBP may not have reached significance due to the variability that is often observed in DBPs within an individual [34]. For this 
Table 7. Genotype class specific mean values for the diabetes and metabolic phenotypes that were associated with common SNPs

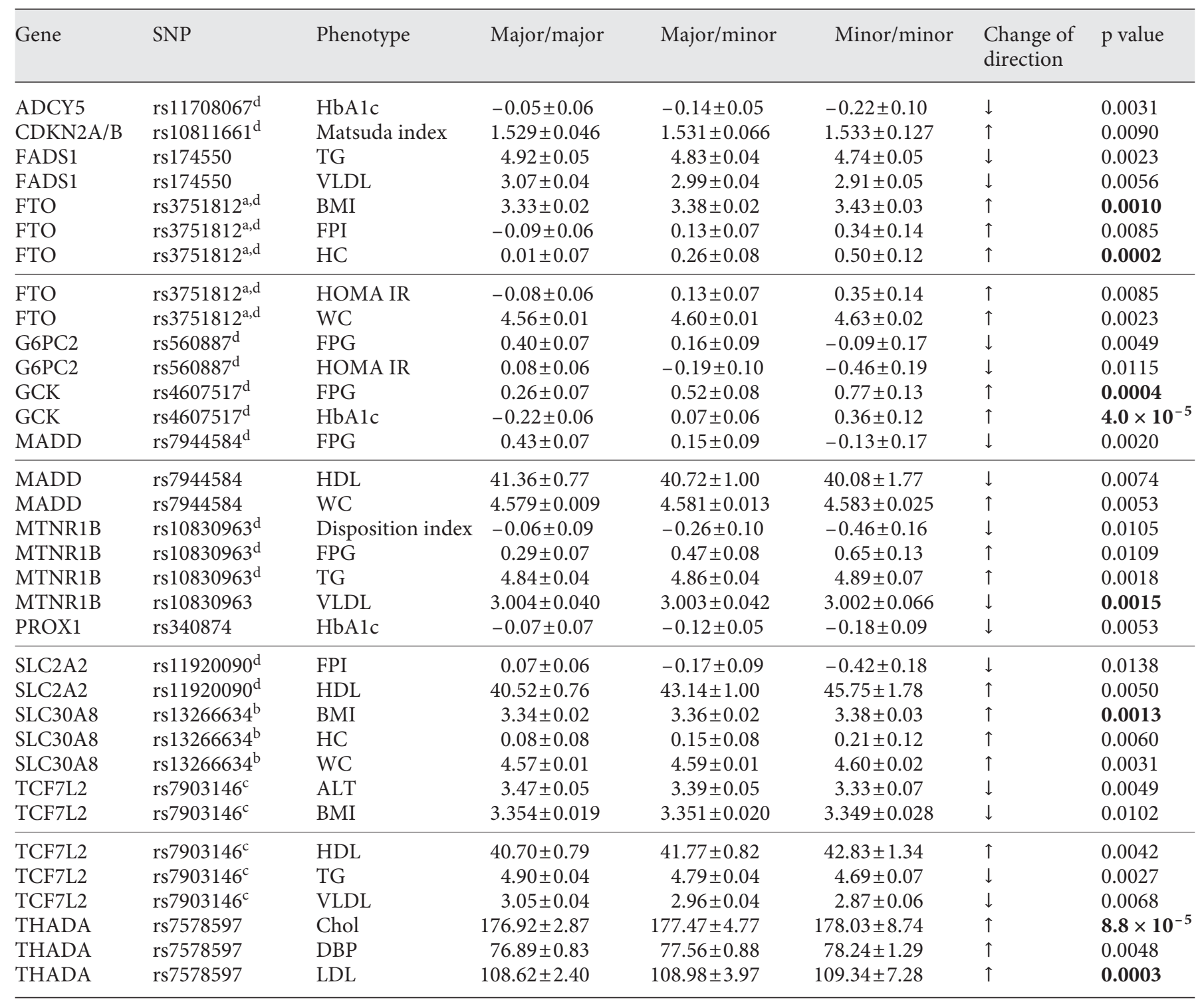

Both corrected ( $\mathrm{p} \leq 0.0015$; in bold) and uncorrected ( $\mathrm{p} \leq 0.01) \mathrm{p}$ values for multiple testing by trait are shown; for HbA1c, HC, FPI, HOMA IR, FPG and disposition index, inverse normalized values are shown; for TG, VLDL, BMI, WC, Matsuda index and ALT, log transformed values are shown.

a Strong LD with $\mathrm{r}^{2}$ ranging from 0.87 to $0.98 .{ }^{\mathrm{b}}$ Strong LD with $\mathrm{r}^{2}=0.90 .{ }^{\mathrm{c}}$ Strong LD with $\mathrm{r}^{2}$ ranging from 0.86 to $0.99 .{ }^{\mathrm{d}} \mathrm{Of}$ the 34 significant ( $\mathrm{p} \leq 0.01$ ) associations shown, 17 were found to replicate the same direction of association (i.e. same risk allele) as the one identified in the original GWAS or candidate gene studies (online suppl. table 1).

study, every attempt was made to measure DBP appropriately, since we measured DBP twice and calculated the average. However, it has been suggested that repeated measurements increase the precision in assessing an individual's underlying mean pressure and it may have been more appropriate to take additional measurements
[34]. Although FPG, 2hOGTT and prediabetes status heritability estimates were not significant, they were trending towards significance and were within the heritability estimate ranges of other previously published studies [31]. The heritability estimate of diabetes was significantly different from zero, but it was imprecise. There is rela- 
tively little power to calculate heritability for diabetes affection status in this data set, attributable to the age-adjusted prevalence of about $9 \%$. There is considerable loss of power observed for dichotomous versus continuous traits, and this power loss is a function of prevalence [35]. Therefore, the appropriate interpretation is that it appears heritable in this population, but the estimate is imprecise. Overall, the majority of the heritability estimates for the phenotypes tested were significant, indicating that the AIR registry is well suited for the identification of genetic factors contributing to diabetes and metabolic syndrome related traits.

The purpose of this study was 2 -fold in that it allowed us to estimate the heritabilities of the phenotypes collected as part of the AIR registry and to perform genetic association analyses in the sample. Specifically, we performed a common SNP genotype association analysis with type 2 diabetes and metabolic syndrome related traits in the AIR registry. The common SNPs were selected from the first-generation GWAS analyses, which have been reviewed elsewhere and are presented in online supplementary table $1[7,13]$. Our goal was to determine whether an association between the risk alleles with type 2 diabetes and related phenotypes was present in our cohort, since the majority of the first-generation GWAS analyses were performed on European populations [7, 13].

There have been a handful of studies that have performed GWAS in Mexican Americans. A GWAS for the identification of type 2 diabetes susceptibility variants/ genes in Mexican Americans from Starr County, Texas, revealed several SNPs (rs979752 and rs10500641 near UBQLNL and OR52H1 on chromosome 11; rs2773080 and rs3922812 in or near RALGPS2 on chromosome 1, and rs1509957 near EGR2 on chromosome 10), with suggestive evidence for association [36]. More recently, there has been a GWAS and meta-analysis performed on Mexican Americans from Starr County, Texas, and Mexicans living in Mexico City, Mexico [21, 22]. This GWAS in Mexican Americans identified a novel locus (C14orf70) and provided suggestive associations for other previously reported loci (HNF1A, KCNQ1, IGF2BP2 and CDKN2A/CDKN2B) [22]. The meta-analysis top signals included 49 SNPs in 8 gene regions (PER3, PARD3B, EPHA4, TOMM7, PTPRD, HNT, LOC729993 and IL34) and 6 intergenic regions. Lastly, a study involving a Mexican Mestizo population using previously identified type 2 diabetes risk variants demonstrated an association of diabetes with the markers rs13266634 (SLC30A8), rs7923837 (HHEX), rs10811661 (CDKN2A/2B),

Genetic Associations with Diabetes and Metabolic Traits rs4402960 (IGF2BP2), rs12779790 (CDC123/CAMK1D) and rs2237892 (KCNQ1) [23].

We identified 20 SNPs nominally associated with one or more of the diabetes related traits $(\mathrm{p} \leq 0.05)$ and 25 SNPs with one or more of the metabolic phenotypes tested. Of note, 5 SNPs from 5 genes (BMI, HC: rs3751812/ FTO; FPG, HbA1c: rs4607517/GCK; VLDL: rs10830963/ MTNR1B; BMI: rs13266634/SLC30A8, and Chol, LDL: rs7578597/THADA) were significantly associated ( $\mathrm{p} \leq$ 0.0015 ) with obesity, glycemic and lipid phenotypes when adjusting for multiple testing using the method of $\mathrm{Li}$ and Ji [30]. Several correlated traits were examined in our study; however, additional correcting for multiple testing with regard to multiple correlated traits is not straightforward, given that the models and traits are not independent $[1,37,38]$.

We found relatively strong associations for the FTO SNP rs3751812 with obesity measures. FTO is a fat mass and obesity-associated protein, but in spite of the name, the function of this protein still remains unclear [39]. This SNP has been highly associated with obesity in humans and, by extension, is related to type 2 diabetes $[39,40]$. The strong association of FTO with obesity measures has been demonstrated in our cohort with the deleterious minor allele T associating with BMI, HC and WC. Our findings are in agreement with previously published studies that have shown similar results indicating that the minor SNP allele of rs3751812 conferred a predisposition to obesity [39]. Moreover, the minor allele of this SNP was associated with HOMA IR and FPI, indicating that this variant in our Mexican American population is also associated with diabetes related traits.

TCF7L2 codes for a transcription factor involved in the Wnt signaling pathway and has been associated with type 2 diabetes in multiple ethnic groups [41-48]. We found 5 SNPs in TCF7L2 (rs7901695, rs4506565, rs7903146, rs12243326 and rs12255372) that were in high LD, confirming what has been described in other populations [49]. In the present study, the minor alleles of each of these SNPs were associated with higher HDL and lower VLDL, TG, ALT and BMI. In our study, we demonstrated an association with diabetes for rs12255372 in TCF7L2, although the statistical significance was suggestive ( $p=0.055)$. To date, most of the TCF7L2 SNP association studies have demonstrated an association with diabetes and glycemic related phenotypes [13], and it has been suggested that the TCF7L2 SNPs are the strongest known genetic risk factor for type 2 diabetes [45].

The study in the Mexican Mestizo population using previously identified type 2 diabetes risk variants dem- 
onstrated an association of diabetes with rs13266634 (SLC30A8) [23]. In our study, we did not demonstrate an association with diabetes; however, we did show that the risk allele was associated with higher levels of BMI, HC and WC. This indicates, therefore, that this variant is associated with measures of obesity in our Mexican American population.

GCK (Glucokinase) is an enzyme that regulates glucose cycling in several tissues, including the liver and pancreas [50]. SNP rs4607517 in this gene has been implicated with fasting glucose levels and type 2 diabetes in multiple populations, making it an excellent candidate to explore in our population $[51,52]$. In the present study, we observed associations with the minor allele A and elevated FPG and HbAlc. These findings of elevated FPG levels with the minor allele A correspond to a previous observation in another Mexican American population study, further validating the strength of the associations we reported [50].

THADA (thyroid adenoma associated) is known to lower measures of $\beta$-cell function [53]. This is further supported by the fact that numerous associations between SNP rs7578597 in THADA and type 2 diabetes related traits have been observed in other populations [54, 55]. In our Mexican American population, we observed a nominal association with prediabetes, reaffirming the previous findings in other studies. Moreover, we observed strong associations with Chol and LDL, illustrating the multifactorial nature of the metabolic syndrome.

Although our discussion has focused mainly on those SNPs that are relatively strongly associated with multiple phenotypes, we nominally replicated other GWAS SNPs previously reported with diabetes or metabolic phenotypes, including ADCY5, CDKN2A/B, CRY2, FADS1,
G6PC2, GLIS3, HNF1B, IGF1, IGF2BP2, MADD, MTNR1B, JAZF1, PROX1 and SLC2A2. Together, our results demonstrate multiple common GWAS SNP associations with diabetes and metabolic-syndrome traits in the AIR registry. These validation findings of multiple SNP associations with diabetes and metabolic syndrome related traits demonstrate that these SNPs contribute to the genetics of these complex diseases in our cohort. The results of this study are promising, though further work needs to be performed. Larger cohorts of Mexican Americans are required to test these SNPs further and to better understand how genetic factors contribute to diabetes and the metabolic syndrome in this high-risk population. Several of the associations found in our study are replications of what has previously been observed in other populations, but there were also notable associations that were not similarly replicated in this study. This could infer a potential genetic difference between our cohort and other study populations, or it may be attributed to the sample size available for this study.

\section{Acknowledgements}

We thank the participants of the AIR registry and are grateful for their participation and cooperation. We thank Veronica Vital, $\mathrm{RN}$, and the ASU clinical research staff for taking excellent care of the participants. We thank Darwin Tsinajinnie and Martha Nunez for their technical assistance.

\section{Disclosure Statement}

The authors do not have any conflicts of interest with respect to the study.

\section{References}

1 Richardson DK, Schneider J, Fourcaudot MJ, Rodriguez LM, Arya R, Dyer TD, Almasy L, Blangero J, Stern MP, Defronzo RA, Duggirala $\mathrm{R}$, Jenkinson $\mathrm{CP}$ : Association between variants in the genes for adiponectin and its receptors with insulin resistance syndrome (IRS)-related phenotypes in Mexican Americans. Diabetologia 2006;49:2317-2328.

- Kashyap SR, Defronzo RA: The insulin resistance syndrome: physiological considerations. Diab Vasc Dis Res 2007;4:13-19.

3 Flegal KM, Carroll MD, Kit BK, Ogden CL: Prevalence of obesity and trends in the distribution of body mass index among us adults, 1999-2010. JAMA 2012;307:491-497.
4 Ford ES, Li C, Zhao G: Prevalence and correlates of metabolic syndrome based on a harmonious definition among adults in the US. J Diabetes 2010;2:180-193.

5 Temelkova-Kurktschiev T, Stefanov T: Lifestyle and genetics in obesity and type 2 diabetes. Exp Clin Endocrinol Diabetes 2012;120: $1-6$.

6 Ahlqvist E, Ahluwalia TS, Groop L: Genetics of type 2 diabetes. Clin Chem 2011;57:241254.

7 Dupuis J, Langenberg C, Prokopenko I, et al: New genetic loci implicated in fasting glucose homeostasis and their impact on type 2 diabetes risk. Nat Genet 2010;42:105-116. $\checkmark 8$ Elbein SC: Perspective: the search for genes for type 2 diabetes in the post-genome era. Endocrinology 2002;143:2012-2018.

-9 Gloyn AL, McCarthy MI: The genetics of type 2 diabetes. Best Pract Res Clin Endocrinol Metabol 2001;15:293-308.

10 Horenstein RB, Shuldiner AR: Genetics of diabetes. Rev Endocri Metab Disord 2004;5:2536.

11 McCarthy MI: Growing evidence for diabetes susceptibility genes from genome scan data. Curr Diab Rep 2003;3:159-167.

-12 Mercado MM, McLenithan JC, Silver KD, Shuldiner AR: Genetics of insulin resistance. Curr Diab Rep 2002;2:83-95. 
13 Prokopenko I, McCarthy MI, Lindgren CM: Type 2 diabetes: new genes, new understanding. Trends Genet 2008;24:613-621.

-14 Diabetes Genetics Initiative of Broad Institute of Harvard and MIT, Lund University, and Novartis Institutes of BioMedical Research, Saxena R, Voight BF, Lyssenko V, et al: Genome-wide association analysis identifies loci for type 2 diabetes and triglyceride levels. Science 2007;316:1331-1336.

$\checkmark 15$ Hanson RL, Bogardus C, Duggan D, Kobes S, Knowlton $\mathrm{M}$, Infante $\mathrm{AM}$, Marovich $\mathrm{L}$, Benitez D, Baier LJ, Knowler WC: A search for variants associated with young-onset type 2 diabetes in American Indians in a $100 \mathrm{~K}$ genotyping array. Diabetes 2007;56:3045-3052.

16 Scott LJ, Mohlke KL, Bonnycastle LL, et al: A genome-wide association study of type 2 diabetes in Finns detects multiple susceptibility variants. Science 2007;316:1341-1345.

17 Sladek R, Rocheleau G, Rung J, Dina C, Shen L, Serre D, Boutin P, Vincent D, Belisle A, Hadjadj S, Balkau B, Heude B, Charpentier G, Hudson TJ, Montpetit A, Pshezhetsky AV, Prentki M, Posner BI, Balding DJ, Meyre D, Polychronakos C, Froguel P: A genome-wide association study identifies novel risk loci for type 2 diabetes. Nature 2007;445:881-885.

18 Zeggini E, Scott LJ, Saxena R, et al: Meta-analysis of genome-wide association data and large-scale replication identifies additional susceptibility loci for type 2 diabetes. Nat Genet 2008;40:638-645.

-19 Zeggini E, Weedon MN, Lindgren CM, et al: Replication of genome-wide association signals in UK samples reveals risk loci for type 2 diabetes. Science 2007;316:1336-1341.

20 Sanghera DK, Blackett PR: Type 2 diabetes genetics: beyond GWAS. J Diabetes Metab 2012; 3:6948.

- 21 Below JE, Gamazon ER, Morrison JV, Konkashbaev A, Pluzhnikov A, McKeigue PM, Parra EJ, Elbein SC, Hallman DM, Nicolae DL, Bell GI, Cruz M, Cox NJ, Hanis CL: Genome-wide association and meta-analysis in populations from Starr County, Texas, and Mexico City identify type 2 diabetes susceptibility loci and enrichment for expression quantitative trait loci in top signals. Diabetologia 2011;54:2047-2055.

22 Parra EJ, Below JE, Krithika S, Valladares A, Barta JL, Cox NJ, Hanis CL, Wacher N, Garcia-Mena J, Hu P, Shriver MD; Diabetes Genetics Replication Meta-analysis (DIAGRAM) Consortium, Kumate J, McKeigue PM, Escobedo J, Cruz M: Genome-wide association study of type 2 diabetes in a sample from Mexico City and a meta-analysis of a Mexican-American sample from Starr County, Texas. Diabetologia 2011;54:2038-2046.

-23 Gamboa-Melendez MA, Huerta-Chagoya A, Moreno-Macias H, et al: Contribution of common genetic variation to the risk of type 2 diabetes in the Mexican Mestizo population. Diabetes 2012;61:3314-3321.
Lehman DM, Hunt KJ, Leach RJ, Hamlington J, Arya R, Abboud HE, Duggirala R, Blangero J, Goring HH, Stern MP: Haplotypes of transcription factor 7-like 2 (TCF7L2) gene and its upstream region are associated with type 2 diabetes and age of onset in Mexican Americans. Diabetes 2007;56:389-393.

25 Shaibi GQ, Coletta DK, Vital V, Mandarino LJ: The design and conduct of a communitybased registry and biorepository: A focus on cardiometabolic health in latinos. Clin Transl Sci 2013;6:429-434.

26 Kim JY, Coletta DK, Mandarino LJ, Shaibi GQ: Glucose response curve and type 2 diabetes risk in Latino adolescents. Diabetes Care 2012;35:1925-1930.

27 Almasy L, Blangero J: Multipoint quantitative-trait linkage analysis in general pedigrees. Am J Hum Genet 1998;62:1198-1211.

28 Boerwinkle E, Chakraborty R, Sing CF: The use of measured genotype information in the analysis of quantitative phenotypes in man. I. Models and analytical methods. Ann Hum Genet 1986;50:181-194.

29 Abecasis GR, Cookson WO, Cardon LR: Pedigree tests of transmission disequilibrium. Eur Hum Genet 2000;8:545-551.

30 Li J, Ji L: Adjusting multiple testing in multilocus analyses using the eigenvalues of a correlation matrix. Heredity 2005;95:221-227.

31 Jowett JB, Diego VP, Kotea N, Kowlessur S, Chitson P, Dyer TD, Zimmet P, Blangero J: Genetic influences on type 2 diabetes and metabolic syndrome related quantitative traits in mauritius. Twin Res Hum Genet 2009; 12:44-52.

-32 Cowie CC, Rust KF, Byrd-Holt DD, Gregg EW, Ford ES, Geiss LS, Bainbridge KE, Fradkin JE: Prevalence of diabetes and high risk for diabetes using A1C criteria in the U.S. population in 1988-2006. Diabetes Care 2010;33: 562-568.

33 Cowie CC, Rust KF, Ford ES, Eberhardt MS, Byrd-Holt DD, Li C, Williams DE, Gregg EW, Bainbridge KE, Saydah SH, Geiss LS: Full accounting of diabetes and pre-diabetes in the U.S. population in 1988-1994 and 20052006. Diabetes Care 2009;32:287-294.

34 Hughes MD, Pocock SJ: Within-subject diastolic blood pressure variability: Implications for risk assessment and screening. J Clin Epidemiol 1992;45:985-998.

35 Williams JT, Blangero J: Power of variance component linkage analysis - II. Discrete traits. Ann Hum Genet 2004;68:620-632.

36 Hayes MG, Pluzhnikov A, Miyake K, Sun Y, $\mathrm{Ng}$ MC, Roe CA, Below JE, Nicolae RI, Konkashbaev A, Bell GI, Cox NJ, Hanis CL: Identification of type 2 diabetes genes in Mexican Americans through genome-wide association studies. Diabetes 2007;56:3033-3044.

37 Coletta DK, Schneider J, Stern MP, Blangero J, DeFronzo RA, Duggirala R, Jenkinson CP: Association of neuropeptide Y receptor Y5 polymorphisms with dyslipidemia in Mexican Americans. Obesity 2007;15:809-815.
38 Jenkinson CP, Coletta DK, Flechtner-Mors M, Hu SL, Fourcaudot MJ, Rodriguez LM, Schneider J, Arya R, Stern MP, Blangero J, Duggirala R, DeFronzo RA: Association of genetic variation in ENPP1 with obesity-related phenotypes. Obesity 2008;16:17081713.

39 Frayling TM, Timpson NJ, Weedon MN, et al: A common variant in the FTO gene is associated with body mass index and predisposes to childhood and adult obesity. Science 2007; 316:889-894.

40 Villalobos-Comparan M, Teresa FloresDorantes M, Teresa Villarreal-Molina M, et al: The FTO gene is associated with adulthood obesity in the Mexican population. Obesity 2008;16:2296-2301.

41 Scott LJ, Bonnycastle LL, Willer CJ, Sprau AG, Jackson AU, Narisu N, Duren WL, Chines PS, Stringham HM, Erdos MR, Valle TT, Tuomilehto J, Bergman RN, Mohlke KL, Collins FS, Boehnke M: Association of transcription factor 7-like 2 (TCF7L2) variants with type 2 diabetes in a Finnish sample. Diabetes 2006;55:2649-2653.

42 Sale MM, Smith SG, Mychaleckyj JC, Keene KL, Langefeld CD, Leak TS, Hicks PJ, Bowden DW, Rich SS, Freedman BI: Variants of the transcription factor 7-like 2 (TCF7L2) gene are associated with type 2 diabetes in an African-American population enriched for nephropathy. Diabetes 2007;56:2638-2642.

43 Miyake K, Horikawa Y, Hara K, Yasuda K, Osawa H, Furuta H, Hirota Y, Yamagata K, Hinokio Y, Oka Y, Iwasaki N, Iwamoto Y, Yamada Y, Seino Y, Maegawa H, Kashiwagi A, Yamamoto K, Tokunaga K, Takeda J, Makino H, Nanjo K, Kadowaki T, Kasuga M: Association of TCF7L2 polymorphisms with susceptibility to type 2 diabetes in 4,087 Japanese subjects. J Hum Genet 2008;53:174-180.

-44 Groves CJ, Zeggini E, Minton J, Frayling TM, Weedon MN, Rayner NW, Hitman GA, Walker M, Wiltshire S, Hattersley AT, McCarthy MI: Association analysis of 6,736 U.K. subjects provides replication and confirms TCF7L2 as a type 2 diabetes susceptibility gene with a substantial effect on individual risk. Diabetes 2006;55:2640-2644.

45 Grant SF, Thorleifsson G, Reynisdottir I, et al: Variant of transcription factor 7-like 2 (TCF7L2) gene confers risk of type 2 diabetes. Nat Genet 2006;38:320-323.

46 Chang YC, Chang TJ, Jiang YD, Kuo SS, Lee KC, Chiu KC, Chuang LM: Association study of the genetic polymorphisms of the transcription factor 7-like 2 (TCF7L2) gene and type 2 diabetes in the Chinese population. $\mathrm{Di}$ abetes 2007;56:2631-2637.

47 Chandak GR, Janipalli CS, Bhaskar S, Kulkarni SR, Mohankrishna P, Hattersley AT, Frayling TM, Yajnik CS: Common variants in the TCF7L2 gene are strongly associated with type 2 diabetes mellitus in the Indian population. Diabetologia 2007;50:63-67. 
48 Cauchi S, Meyre D, Dina C, Choquet H, Samson C, Gallina S, Balkau B, Charpentier G, Pattou F, Stetsyuk V, Scharfmann R, Staels B, Fruhbeck G, Froguel P: Transcription factor TCF7L2 genetic study in the French population: expression in human beta-cells and adipose tissue and strong association with type 2 diabetes. Diabetes 2006;55:2903-2908.

-49 Kang ES, Kim MS, Kim YS, Hur KY, Han SJ, Nam CM, Ahn CW, Cha BS, Kim SI, Lee HC: A variant of the transcription factor 7-like 2 (TCF7L2) gene and the risk of posttransplantation diabetes mellitus in renal allograft recipients. Diabetes Care 2008;31:63-68.

50 Li X, Shu YH, Xiang AH, Trigo E, Kuusisto J, Hartiala J, Swift AJ, Kawakubo M, Stringham HM, Bonnycastle LL, Lawrence JM, Laakso M, Allayee H, Buchanan TA, Watanabe RM: Additive effects of genetic variation in GCK and G6PC2 on insulin secretion and fasting glucose. Diabetes 2009;58:2946-2953.
51 Chambers JC, Zhang W, Zabaneh D, Sehmi J, Jain P, McCarthy MI, Froguel P, Ruokonen A, Balding D, Jarvelin MR, Scott J, Elliott P, Kooner JS: Common genetic variation near melatonin receptor MTNR1B contributes to raised plasma glucose and increased risk of type 2 diabetes among Indian Asians and European Caucasians. Diabetes 2009;58:27032708.

52 Fujita H, Hara K, Shojima N, Horikoshi M, Iwata M, Hirota Y, Tobe K, Seino S, Kadowaki T: Variations with modest effects have an important role in the genetic background of type 2 diabetes and diabetes-related traits. J Hum Genet 2012;57:776-779.

53 Simonis-Bik AM, Nijpels G, van Haeften TW, Houwing-Duistermaat JJ, Boomsma DI, Reiling E, van Hove EC, Diamant M, Kramer MH, Heine RJ, Maassen JA, Slagboom PE, Willemsen G, Dekker JM, Eekhoff EM, de Geus EJ, 't Hart LM: Gene variants in the novel type 2 diabetes loci CDC123/CAMK1D, THADA, ADAMTS9, BCL11A, and MTNR1B affect different aspects of pancreatic beta-cell function. Diabetes 2010;59:293301.
4 Gupta V, Vinay DG, Rafiq S, Kranthikumar MV, Janipalli CS, Giambartolomei C, Evans DM, Mani KR, Sandeep MN, Taylor AE, Kinra S, Sullivan RM, Bowen L, Timpson NJ, Smith GD, Dudbridge F, Prabhakaran D, Ben-Shlomo Y, Reddy KS, Ebrahim S, Chandak GR; Indian Migration Study Group: Association analysis of 31 common polymorphisms with type 2 diabetes and its related traits in Indian sib pairs. Diabetologia 2012; 55:349-357.

$55 \mathrm{Hu}$ C, Zhang R, Wang C, Wang J, Ma X, Lu J, Qin W, Hou X, Wang C, Bao Y, Xiang K, Jia W: PPARG, KCNJ11, CDKAL1, CDKN2ACDKN2B, IDE-KIF11-HHEX, IGF2BP2 and SLC30A8 are associated with type 2 diabetes in a chinese population. PLoS One 2009;4: e7643.

\section{Erratum}

In the article by Farook VS et al., entitled 'Linkage of type 2 diabetes on chromosome 9p24 in Mexican Americans: additional evidence from the Veterans Administration Genetic Epidemiology Study (VAGES)' [Hum Hered 2013;76:36-46, DOI:10.1159/000354849], the name of the institute has changed from 'Southwest Foundation for Biomedical Research, San Antonio, Tex.' to 'Texas Biomedical Research Institute, San Antonio, Tex.'. 